\title{
Anterior Cruciate Ligament Injury: Compensation during Gait using Hamstring Muscle Activity
}

\author{
Paola Formento Catalfamo*, Gerardo Aguiar, Jorge Curi and Ariel Braidot
}

Department of Biomechanics, School of Engineering, National University of Entre Rios, Argentina

\begin{abstract}
Previous research has shown that an increase in hamstring activation may compensate for anterior tibial transalation (ATT) in patients with anterior cruciate ligament deficient knee (ACLd); however, the effects of this compensation still remain unclear. The goals of this study were to quantify the activation of the hamstring muscles needed to compensate the ATT in ACLd knee during the complete gait cycle and to evaluate the effect of this compensation on quadriceps activation and joint contact forces. A two dimensional model of the knee was used, which included the tibiofemoral and patellofemoral joints, knee ligaments, the medial capsule and two muscles units. Simulations were conducted to determine the ATT in healthy and ACLd knee and the hamstring activation needed to correct the abnormal ATT to normal levels (100\% compensation) and to $50 \%$ compensation. Then, the quadriceps activation and the joint contact forces were calculated. Results showed that $100 \%$ compensation would require hamstring and quadriceps activations larger than their maximum isometric force, and would generate an increment in the peak contact force at the tibiofemoral (115\%) and patellofemoral (48\%) joint with respect to the healthy knee. On the other hand, 50\% compensation would require less force generated by the muscles (less than 0.85 of maximum isometric force) and smaller contact forces (peak tibiofemoral contact force increased $23 \%$ and peak patellofemoral contact force decreased $7.5 \%$ with respect to the healthy knee). Total compensation of ATT by means of increased hamstring activity is possible; however, partial compensation represents a less deleterious strategy.
\end{abstract}

Keywords: Anterior cruciate ligament, tibial translation, hamstring.

\section{INTRODUCTION}

Injury to the Anterior Cruciate Ligament (ACL) is very frequent in sport related environments. Approximately 100.000 skiers suffer an injury to their ACL each year in USA [1]. The main function of the ACL is the prevention of anterior translation of the tibia (ATT) relative to the femur. Normally, an ACL rupture results in loss of knee joint stability, decreased muscle force and reduced functionality, and often requires reconstructive surgery [2].

A large number of in vivo [3-5], in vitro [6,7] and modelling studies $[8,9]$ have shown that hamstring activation may reduce ATT in the ACL deficient (ACLd) knee.

However, only two studies have quantified the hamstring activation needed to compensate ATT. One of them also evaluated the effect of the compensation for one sample of the gait cycle.

Liu and Maitland [10] used a bidimensional model of the knee to examine the effect of various levels of hamstring muscle activation on restraining ATT in the ACLd knee during level walking. Simulations were conducted for a single selected position of the gait cycle, during early stance phase of gait. The results showed that the ATT increased by $11.8 \mathrm{~mm}$ in ACLd knee, while $56 \%$ of the maximal hamstring muscle force could reduce it to a normal level.

*Address correspondence to this author at the Department of Biomechanics, School of Engineering, National University of Entre Ríos, Ruta 11. Km 10, Oro Verde, Argentina, Tel: 0054-343-4975100 ext: 121; Fax: 0054-343 4975100 ext: 108; E-mail: pcatalfamo@bioingenieria.edu.ar
The consequences of increased hamstring muscle force included increased quadriceps muscle force and joint contact forces. In fact, when hamstring activation was applied to 56 $\%$ of its maximal isometric force (MIF), the force generated by the quadriceps muscle increased $86.5 \%$ with respect to normal knee, while tibiofemoral contact force increased 118 $\%$ and patellofemoral contact force increased approximately $100 \%$.

Shelburne et al. [11] used a three dimensional model of the lower limb to determine whether an isolated change in either quadriceps or hamstrings muscle force was sufficient to stabilize the ACLd knee. The simulations considered either a hamstring facilitation (increasing the force generated by the muscles) or quadriceps avoidance (decreasing the force generated by them) pattern. Simulations in this case were conducted for 23 points that represented the whole gait cycle. The results showed that increased hamstrings force (20\% of maximal hamstring muscle force) was sufficient to stabilize the ACLd knee while reduced quadriceps force was insufficient to stabilize it during the complete gait cycle.

Increased hamstring muscle forces may produce an additional knee flexion moment that must be balanced by a corresponding increase in quadriceps muscle force [10]. An increase in co-contraction of antagonist muscles would decrease motion efficiency, increase energy expenditure and augment tibiofemoral contact force.

Also, the loading within musculoskeletal structure influences biological processes including fracture healing [12], bone remodelling [13] and ligament repair [14]; it is also believed to play a role in the onset of orthoarthritis [15]. 
It is important then to understand the changes in quadriceps force and joint loading provoked by increased hamstring force.

The objective of the current study was to calculate the hamstring activation needed to correct the abnormal ATT during the whole gait cycle (70 samples) and evaluate the effects of the correction on quadriceps activation and contact force.

\section{METHODS}

The model used in this study is a bidimensional model of the knee in the sagittal plane (Fig. 1) and it is based on the model used by Liu and Maitland [10].

The model included three rigid bodies: the femur, tibia and patella; four ligaments: the anterior and posterior cruciate ligament, medial and lateral collateral ligament; two muscle groups: hamstrings and quadriceps; the patellar tendon and the medial capsule.

Coordinate systems were attached to the tibia and femur (Fig. 1). The position and orientation of the femur relative to the tibia was described in the tibial system by a vector $\left(\mathrm{X}_{0}\right.$, $\left.Y_{0}, \alpha\right)$ that defined the origin of the femoral system and the knee angle [16]. A set of normal gait data [17] was used in the simulations. The knee angle varied from $-3^{\circ}$ of extension to $66.6^{\circ}$ of flexion.

The contour of the tibial plateau was represented by a straight line sloped $8^{\circ}$ posteriorly relative to the $\mathrm{x}$-axis of the tibia coordinate system [18]. The patella was approximated as a rectangle $[19,20]$. From the data presented by Garg and Walker [21] who digitized parasagittal sections of the distal femur, proximal tibia and patella for 23 cadaveric knees, approximating the tibial plateau and patellar facet as flat surfaces seems justifiable in view of the shapes of parasagittal sections taken through the medial or lateral condyle of the tibia and the patella. However, at large knee flexion angles (larger than $90^{\circ}$ ) contact between the patella and femur is not confined to the region of the femoral groove [22]. The normal data set used for simulation in the current study presented knee angles smaller than $70^{\circ}$ during the entire gait cycle. Yet, at flexion angles larger than $90^{\circ}$ our model may not be valid.

The femoral contour of the patellofemoral joint and the contour of the femoral condyle in the tibiofemoral joint were represented by ellipses [20]. The contact surfaces of the tibiofemoral and patellofemoral joints were assumed to be rigid and frictionless. Assuming that the bones are rigid, tibiofemoral and patellofemoral contact occur at single points in the model. However, in the real knee the menisci and cartilage ensure that contact occurs over a finite area at each of these joints. The forces developed by the modeled ligaments, then, would presumably be lower if joint compliance was taken into account. Calculations based on the assumption of rigid contact are therefore likely to overestimate ligament forces in the intact knee.

The contact forces of the joints were along the normal direction of the contact plane at the contact point.

Each ligament was modelled as an elastic element with a non-linear force-strain relationship at low strain levels and a linear force-strain relationship at higher levels [23, 24]. The strain on the ligaments was calculated using data from: a) the position of the insertion points, b) the relative position between femur and tibia $\left(\mathrm{X}_{0}, \mathrm{Y}_{0}, \alpha\right)$ and $\left.\mathrm{c}\right)$ their reference strain (the threshold between the linear and non - linear area). The magnitude of the force in each ligament was expressed as [16].
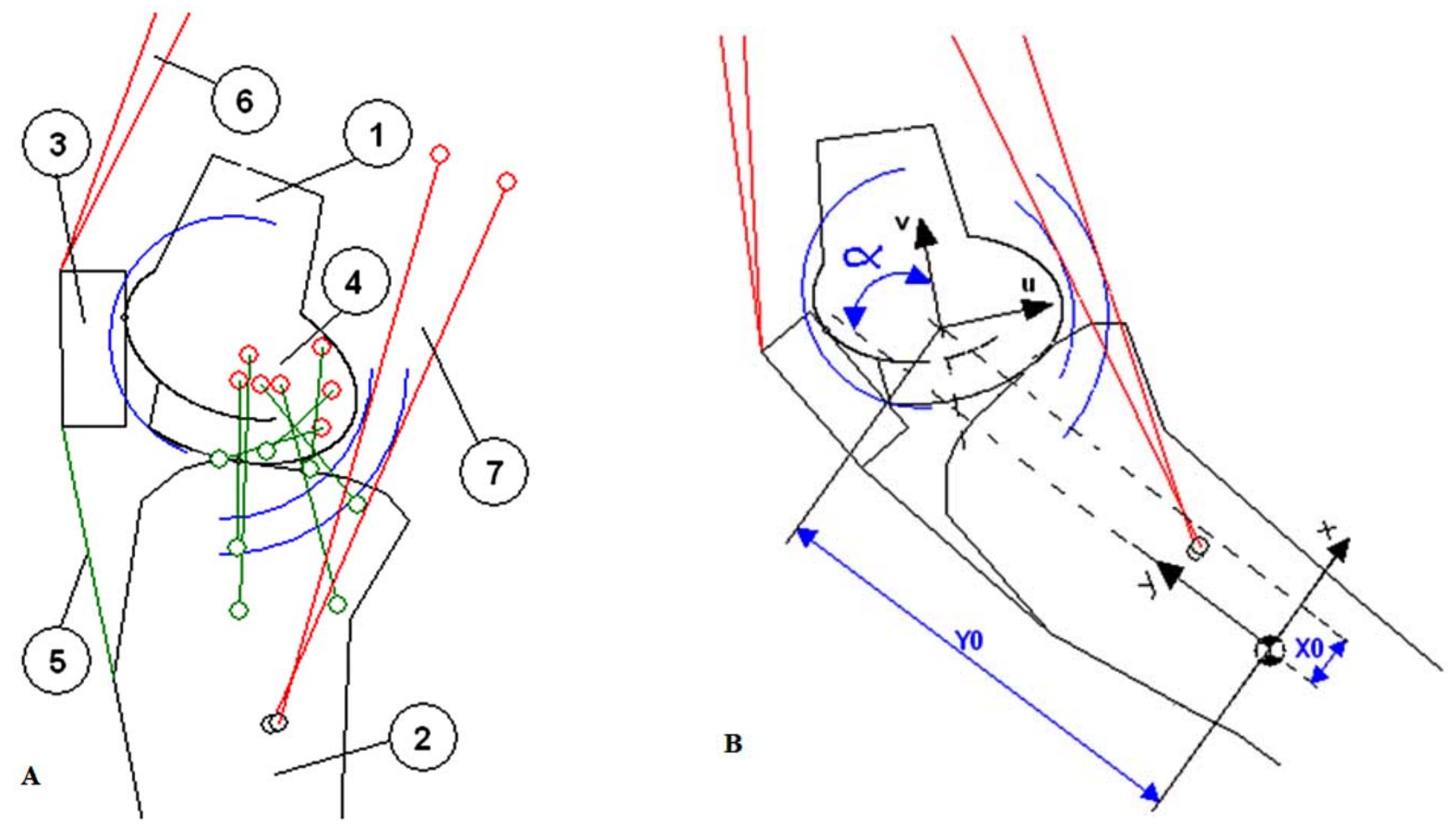

Fig. (1). A: Bidimensional model of the knee used for simulations. 1: Femur, 2: Tibia, 3: Patella, 4: Ligaments of the tibiofemoral contact, 5: patellar tendon; 6: quadriceps muscle, 7: hamstring muscle. B: Coordinate systems were attached to the tibia (x,y) and to the femur (u,v). The position and orientation of the femur relative to the tibia was described in the tibial system using the vector $\left(\mathrm{X}_{0}, \mathrm{Y}_{0}, \alpha\right)$. 
Table 1. Stiffness Coefficients Used for Each of the Ligaments Modelled in this Study. K1: Stiffness Used within Parabolic Region; K2: Stiffness Used within Linear Region

\begin{tabular}{|c|c|c|}
\hline Ligament & $\mathrm{K} 1\left[\mathbf{N ~ \mathbf { ~ m m } ^ { - 1 } ]}\right.$ & $\mathrm{K} 2\left[\mathrm{~N} \mathrm{~mm}^{-2}\right]$ \\
\hline Posterior bundle of the Anterior Cruciate L. & 83.15 & 26.27 \\
\hline Anterior bundle of the Posterior Cruciate L. & 125 & 31.26 \\
\hline Posterior bundle of the Posterior Cruciate L. & 60 & 29.19 \\
\hline Medial Capsule & 52.59 & 12 \\
\hline
\end{tabular}

$F_{j}=\left\{\begin{array}{cc}0 & \text { if } \varepsilon_{j} \leq 0 \\ K_{2 j}\left(L_{j}-L_{0 j}\right)^{2} & \text { if } 0=\varepsilon_{j} \leq 2 \varepsilon_{r e f} ; \\ K_{2 j}\left[L_{j}-\left(1+\varepsilon_{r e f}\right) L_{0 j}\right] & \text { if } \varepsilon_{j}>2 \varepsilon_{r e f} ;\end{array}\right\}$

Where $\varepsilon_{\mathrm{j}}$ is the strain in the jth ligament, $\mathrm{K} 1$ and $\mathrm{K} 2$ are the stiffness coefficients for the jth ligament for the parabolic and linear region, respectively, $\mathrm{L}_{\mathrm{j}}$ is the current length of the ligament and $\mathrm{L}_{\mathrm{oj}}$ is the original length. The reference strain was defined as $\varepsilon_{\text {ref }}=0.03$. Information about the insertion points was obtained from [16, 23]. Table 1 shows the values used for K1 and K2 [16].

The patellar tendon was assumed to be non-extensible due to its high stiffness coefficient (4405 $\mathrm{Nmm}^{-1}$ [25]).

A linear relationship between muscle activation and force produced (proportional to the MIF) was assumed [26].

Muscle and ligament forces acted along the lines that connected their origin sites with their insertion sites. An action circle was used for the hamstrings [27, 28].

The models proposed by Liu and Maitland and Shelburne et al. $[10,11]$ neglected the effects of inertia and centrifugal forces acting on the segments by assuming that the leg remained in static equilibrium. The model used in this study considered the actual dynamic equilibrium, instead, since it represents a more real scenario. The shank moment of inertia about point $\mathrm{P}$ (defined at the intersection between the vertical axis of the tibia and the tibial plateau) was calculated as:

$I_{T}=I_{0}+m_{T} * L_{p}^{2}$

Where:

$I_{T}$ : tibia moment of inertia about point $\mathrm{P}$.

$I_{0}$ : tibia moment of inertia about the centre of mass.

$m_{T}$ : mass of the tibia

$L p \quad$ : length between centre of mass and proximal point (P) (data for $L p$ were obtained from [29])

Then, the following equations for the dynamic equilibrium of the tibia were considered (Fig. 2):

$$
\begin{aligned}
& m_{T} \mathbf{g}+\mathrm{RF}_{A}+\mathrm{CF}_{T F}+\sum \mathbf{F}_{H_{i}}+\sum \mathbf{F}_{L_{i}}+\mathbf{F}_{P T}=m_{T} \mathbf{a}_{T} \\
& M_{T}+M R_{A}+M C_{T F}+\sum M_{H_{i}}+\sum M_{L_{i}}+M_{P T}=I_{T} a_{T}
\end{aligned}
$$

Where:

$\boldsymbol{R F}_{A}$ : reaction force of the foot at the ankle.

$\boldsymbol{C F}_{T F}$ : contact force at the tibio femoral joint.

$\boldsymbol{F}_{H i} \quad$ : hamstring muscle force

$\boldsymbol{F}_{L i} \quad$ : forces from the ligaments.

$\boldsymbol{F}_{P T}$ : force from patellar tendon.

$\boldsymbol{a}_{T,} \alpha_{T}:$ linear and angular acceleration of the tibia.

g : acceleration due to gravity.

$m_{T} \quad$ : mass of the tibia,

$I_{T} \quad$ : moment of inertia of the tibia about point $\mathrm{P}$.

$M_{T} \quad$ : moment of force caused by the weight of the tibia.

$M R_{A} \quad$ : resultant moment of the foot at the ankle (which includes the muscular moment and the moment caused by the reaction force $\boldsymbol{R} \boldsymbol{F}_{A}$ ).

$M C_{T F}$ : moment caused by $\boldsymbol{C F}_{T F}$.

$M_{H i} \quad$ : moment caused by $\boldsymbol{F}_{\mathrm{Hi}}$

$M_{L i} \quad$ : moment caused by $\boldsymbol{F}_{L i}$.

$M_{P T} \quad$ : moment caused by $\boldsymbol{F}_{P T}$.

All moments were considered about point $\mathrm{P}$.

The inertial forces and moments of the patella were ignored since the mass of the patella was considered negligible. Forces and moments produced by the patellar tendon, two units from the quadriceps muscles and the patellofemoral contact force were included in the equations of the patella, as follows:

$$
\begin{aligned}
& \mathbf{C F}_{P F}+\sum \mathbf{F}_{Q_{i}}+\mathbf{F}_{P T} \approx \mathbf{0} \\
& M_{P F}+\sum M_{Q_{i}}+M_{P T} \approx 0
\end{aligned}
$$

Where:

$\boldsymbol{C F}_{P F}$ : Contact Force at the patellofemoral joint.

$\boldsymbol{F}_{Q i}:$ Quadriceps muscle force.

$\mathbf{F}_{P T}$ : Force from patellar tendon.

$M_{P F}:$ Moment caused by $\mathbf{C F}_{P F}$.

$M_{Q i}$ : Moment caused by $\mathbf{F}_{Q i}$.

$M_{P T} \quad$ : Moment caused by $\mathbf{F}_{P T}$ 


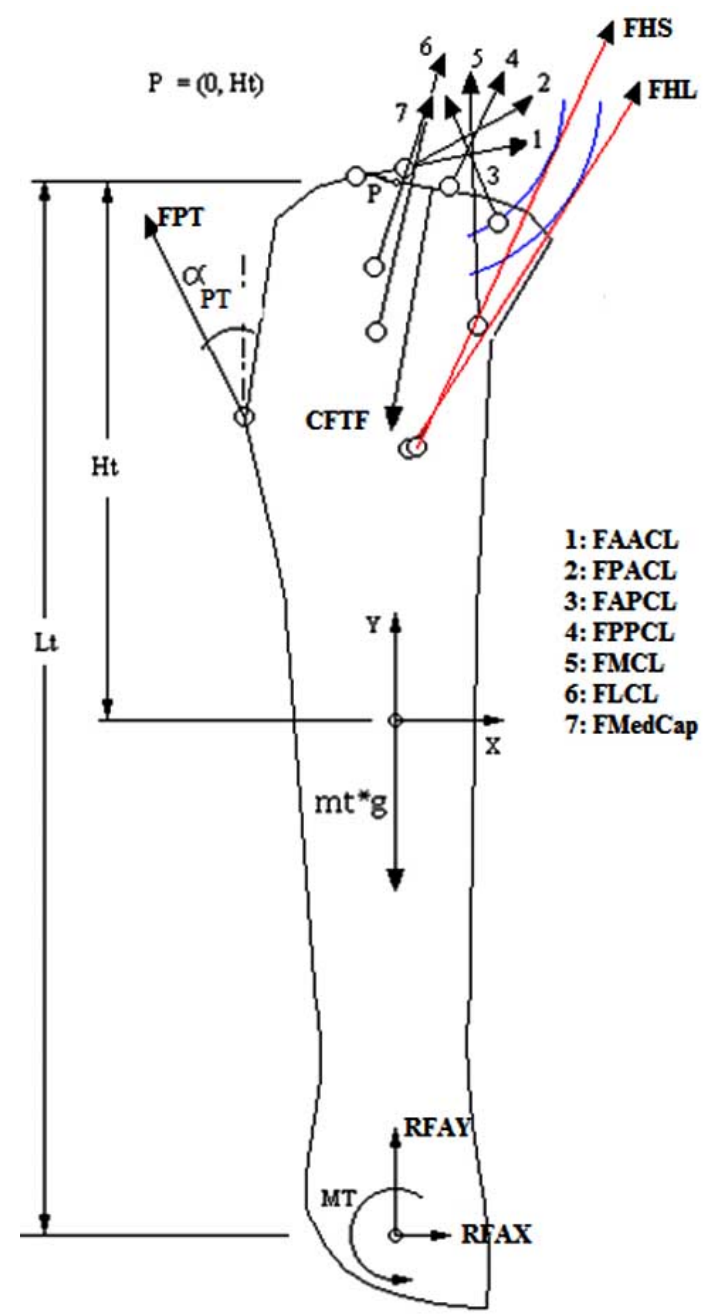

Fig. (2). Forces applied on the tibia include resultant moment (MT) and reaction forces of the foot at the ankle in x (RFAX) and $y$ directions (RFAY), weight of the tibia (mass of the tibia - mt multiplied by gravity $-\mathrm{g}$ ), contact force at the tibio femoral joint (CFTF), and the forces from the patellar tendon (FPT), hamstrings long head (FHL), short head (FHS), anterior bundle of the ACL (FAACL), posterior bundle of the ACL (FPACL), anterior bundle of the PCL (FAPCL), posterior bundle of the PCL (FPPCL), medial collateral ligament (FMCL), lateral collateral ligament (FLCL) and medial capsule (FMedCap). Point P, with coordinates $0, \mathrm{Ht}$, was the reference point to calculate all moments. Lt: length of the tibia; $\alpha_{\mathrm{pt}}$ : angle of patellar tendon.

An iterative algorithm was used to solve the equations of the model for the entire gait cycle:

I. The activation of the hamstring muscle $\left(\mathrm{A}_{\mathrm{H}}\right)$ is defined and the force from the muscle is calculated.

II. An initial value for $\mathrm{X}_{0}$ is proposed.

III. The tibiofemoral contact is solved by calculating:

1. $Y_{0}$, from the knee angle $(\alpha), X_{0}$ and the contact conditions.

2. The orientation and the point of action of the contact force.

3. The orientation and directions of the ligaments, and the force and strain from them.
4. The orientation of the hamstring muscles.

5. The tibiofemoral contact force, the orientation and force from the patellar tendon, from the balance of force and moments of the tibia (equations 1 and 2).

I. Once the orientation of the patellar tendon is known, the coordinates of the bottom vertex of the patella on the anterior face are calculated.

II. The patellofemoral contact is solved, by calculating:

1. The orientation of the patella using a point in the patella (A) and the position of the femur and taking into account the geometric restrictions imposed.

2. The orientation and point of application of the patellofemoral contact force.

3. The orientation of the quadriceps muscles.

4. The patellofemoral contact force and the quadriceps force from the equations of equilibrium of forces in both axes (equation 3 ).

III. The moment of the patella is then calculated (equation 4).

IV. The total moment of the patella should be zero due to its negligible mass, so it is used as error function, for the correction of the value $\mathrm{X}_{0}$ defined in II.

Liu and Maitland [10] also used a set of normal gait data [30] for the simulations. They concentrated the analysis on a single selected position at early stance phase for which they applied incremental hamstring muscle forces ranging from $0 \%$ to $56 \%$ of maximal isometric force.

In the present study, the simulation was expanded to the complete gait cycle. When trying to apply similar range of hamstrings activation, it was noted that the simulation did not converge for the complete range. Minimum values of hamstrings activity were needed so that the geometric and dynamic conditions imposed to the system could be accomplished. These values were found for each sample by incrementing gradually the hamstring activity until the model reached convergence.

Then an activation function for the hamstring was proposed:

$A(t)=A_{0} e^{\left(\frac{t-t_{0}}{100}\right)^{2}}$

Where:

$t \quad$ : time (expressed as percentage of gait cycle),

$t_{0}$ : delay that causes a maximum activation at the end of the gait cycle,

$A_{0}$ : maximal activation, defined as double the maximum activation found for simulation convergence.

The simulations were conducted on the modelled normal and ACLd knees. The ACLd knee was defined as a knee with a completely ruptured ACL so the ACL stiffness coefficient was set to zero [10].

Then, the ATT for healthy and ACLd knees were analysed and the hamstring activation was incremented so 
that the ATT was reduced 50\% and 100\%. Four conditions were considered and analysed (see Table 2).

Table 2. Conditions Considered for Simulation

\begin{tabular}{|c|c|c|c|}
\hline Condition & Knee & Hamstring Activity & ATT Compensation \\
\hline \hline 1 & Normal & Normal & --- \\
\hline 2 & ACLd & Normal & --- \\
\hline 3 & ACLd & Increased & $50 \%$ \\
\hline 4 & ACLd & Increased & $100 \%$ \\
\hline
\end{tabular}

\section{RESULTS}

\section{Minimum Hamstring Activation}

The pattern of minimum hamstring activation required for the complete gait cycle for model convergence is shown in Fig. (3). There were no requirements from the $8 \%$ to the $73 \%$ of the gait cycle.

Values for $A_{0}$ y $t_{0}$ were chosen so that the activation remained greater than the minimum level during the whole cycle. $A_{0}=60 \%$ of MIF and $t_{0}=96 \%$ of gait cycle were selected. Fig. (3) shows the chosen hamstring activation.

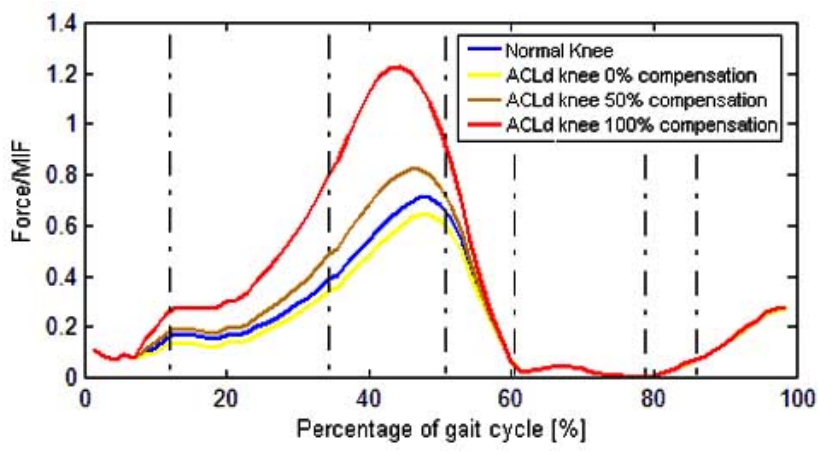

Fig. (3). Forces transmitted to the knee ligaments during normal gait.

The ligament forces in the anterior-posterior direction (relative to the tibial coordinate system) are shown in Fig. (4). No shear force was borne by the anterior bundle of the ACL (aACL) or the posterior bundle of the ACL (pACL) from the $6 \%$ to the $58 \%$ of gait cycle. Peak forces transmitted to the bundles were $450 \mathrm{~N}$ for aACL and $240 \mathrm{~N}$ for pACL and both occurred at $45 \%$ of gait cycle.

The medial capsule (medcap) and the medial collateral ligament (MCL) were loaded during the first half of swing phase (58\% - 82\% of gait cycle); peak forces transmitted to medcap and MCL were much smaller than the one borne by the ACL (48 N and $23 \mathrm{~N}$, respectively).

\section{Healthy Knee and ACLd Knee with and without Compensation}

The ATT for the healthy, ACLd knee, ACLd knee with $50 \%$ and $100 \%$ compensation are shown in Fig. (5).

The peak ATT for the healthy knee was $12 \mathrm{~mm}$ while for ACLd knee reached $19 \mathrm{~mm}$. The ATT in the ACLd knee increased rapidly from the $7 \%$ to the $15 \%$ of gait cycle, reaching a difference of $10 \mathrm{~mm}$ with respect to the ATT in healthy knee, and then remained constant during most of stance phase. During the swing phase of gait, there is a posterior tibial translation at an approximately constant rate (16 $\mathrm{mm}$ change, from $58 \%$ to $86 \%$ of gait cycle).

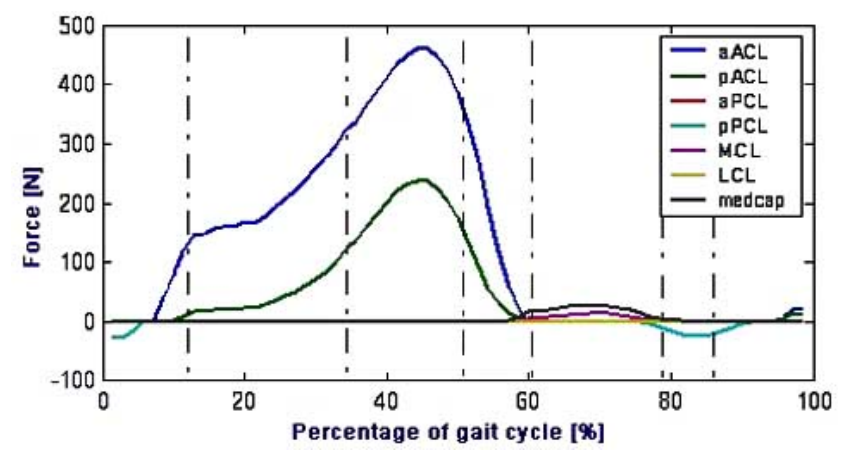

Fig. (4). Ligament forces calculated for the healthy knee (in the anterior direction relative to the tibial coordinate system).

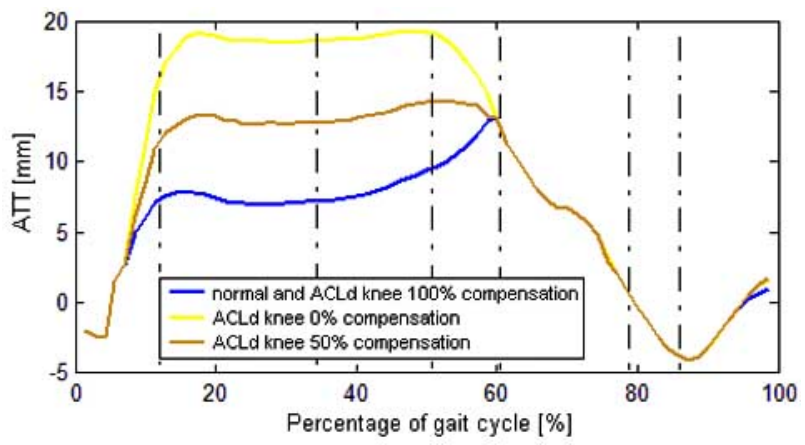

Fig. (5). Anterior Tibial Translation for the healthy and the ACLd knee with $0 \%, 50 \%$ and $100 \%$ compensation.

In order to compensate the abnormal ATT, an increase in the hamstring activation was proposed from the $7 \%$ to the $60 \%$ of gait cycle. Peak hamstring force required for $100 \%$ ATT compensation was $1.35 \mathrm{MIF}$ and occurred at $45 \%$ of gait cycle (Fig. 6). On the other hand, peak hamstring force required for $50 \%$ compensation was 0.4 of MIF and also occurred at $45 \%$ of gait cycle.

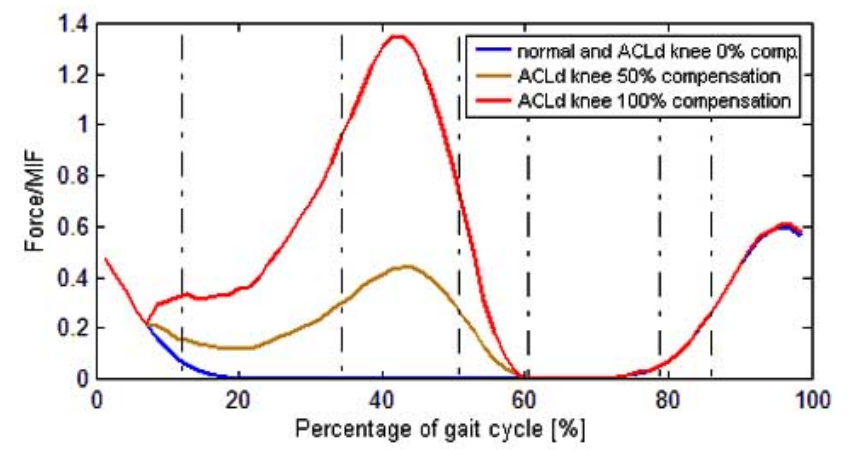

Fig. (6). Hamstring activation for the healthy (normal) and the ACLd knee with 0\%, 50\% and 100\% ATT compensation. 
The tibiofemoral contact force presented a peak value of $4590 \mathrm{~N}$ and $4110 \mathrm{~N}$ for the healthy and ACLd knee, respectively (Fig. 7). Once hamstring compensation was applied, the peak values increased to $5800 \mathrm{~N}$ and $9900 \mathrm{~N}$ for compensations of $50 \%$ and $100 \%$ respectively.

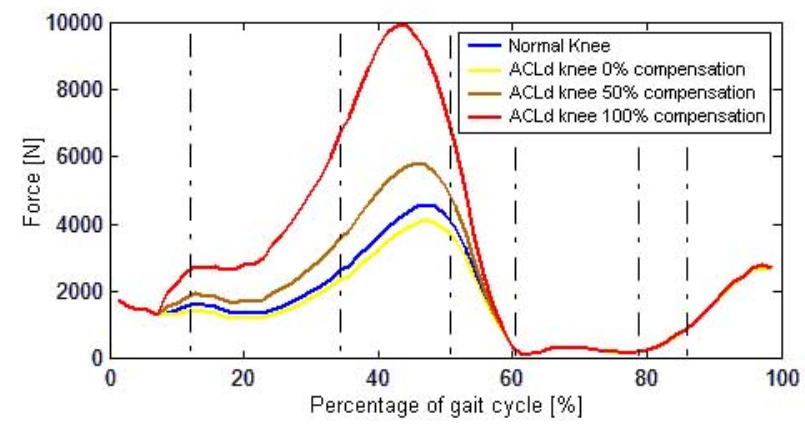

Fig. (7). Tibiofemoral contact force for healthy and ACLd knee with $0 \%, 50 \%$ and $100 \%$ ATT compensation.

Peak value of patellofemoral contact force for the healthy knee and ACLd knee was $1410 \mathrm{~N}$ and $935 \mathrm{~N}$, respectively (Fig. 8). Once compensation was applied, peak patellofemoral force increased to $1305 \mathrm{~N}$ for $50 \%$ and 2085 $\mathrm{N}$ for $100 \%$ (Fig. 8).

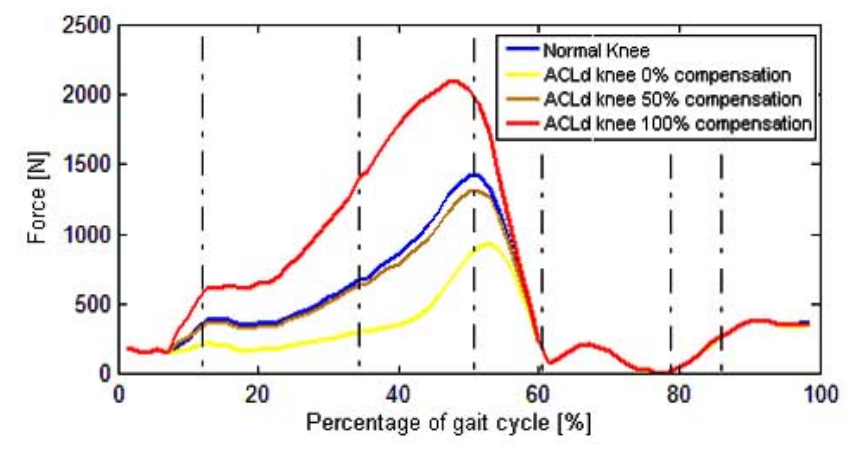

Fig. (8). Patellofemoral contact force for healthy (normal) and ACLd knee with $0 \%, 50 \%$ and $100 \%$ ATT compensation.

Peak force in quadriceps was $0.71 \mathrm{MIF}$ for the healthy knee, 0.64 MIF for ACLd knee, 0.82 MIF for ACLd knee with $50 \%$ of compensation and 1.23 MIF for $100 \%$ compensation (Fig. 9).

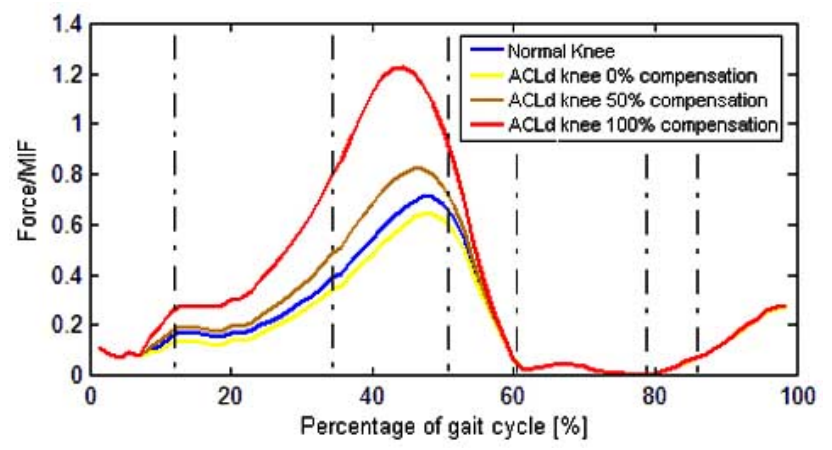

Fig. (9). Quadriceps muscle activation for the healthy (normal) and ACLd knee with $0 \%, 50 \%$ and $100 \%$ ATT compensation.

\section{DISCUSSION}

The model used in this study presented some limitations. First, it simplified the three dimensional anatomical structure of the knee joint to a two dimensional model. Rotations of the knee (estimated to be in the range of $\pm 5^{\circ}$ to $\pm 13^{\circ}[31$, 32]) decrease the force borne by the ACL, which means that predictions of ACL force and ATT may be somewhat overestimated by a bidimensional model [9]. Second, the use of kinematic and kinetic data from a healthy subject to simulate the ACLd knee, represents another limitation. It should be noted that this study focused on the effects of one variable (hamstring activation) on the resultant ATT, quadriceps activation and contact forces. Further investigations should expand this analysis using data from ACLd patients.

The results of this study are in general agreement with previous results reported in the literature. The minimum hamstring activation pattern obtained reflects the pattern reported for normal walking [32-34], suggesting an adequate prediction of muscle activation.

The simulations in this study found that the ACL bore the largest anterior force and it is loaded during stance and unloaded during swing (Fig. 4), in agreement with results presented by Shelburne et al. [34].

Also in agreement with the study by Shelburne [35], the simulation found two peaks of ACL loading for the healthy knee, one during early stance phase (approximately $15 \%$ of gait cycle) and another at toe off (Fig. 5). Similar to the results obtained in vivo by Kvist and Gillquist [36], the simulation showed a peak ATT for healthy knee near toe off.

A wide range of tibiofemoral contact forces have been reported in the literature. Tibiofemoral contact force of 1400 $\mathrm{N}$ (2.5 of body weight -BW) were reported during gait at the $13 \%$ of gait cycle [10]. Peak forces of $3.1 \mathrm{BW}$ for walking [37], 4.3 BW during squat, leg press and knee extension exercises [38] and a variation from 2.7 to $7.5 \mathrm{BW}$ at knee extension angles between $0^{\circ}$ and $40^{\circ}$ at varying angular velocities [39] have been reported. A large number of interindividual factors, such as age and weight [37], activities performed and modelling factors, such as the bony geometry at the knee joint [40] may influence the forces and expand their range. Contact forces calculated by the simulations in the present study are in the order of the ones calculated by Liu and Maitland [10].

The results of this study showed that in order to restore abnormal ATT to normal levels, the hamstring activation should increase to 1.35 of MIF, while the quadriceps activation would increase to 1.23 of MIF. In addition, the peak contact force at the tibiofemoral joint would augment $115 \%$, while the peak force at the patellofemoral joint would increase $48 \%$ in relation to the healthy knee.

On the other hand, a compensation of $50 \%$ of abnormal ATT requires a force generated by the hamstrings of 0.4 of MIF. Interestingly, this activation is smaller than the normal maximum activation of the hamstrings during gait (which occurs during the swing phase and its peak is 0.6 of MIF, see Fig. 6). This compensation causes the quadriceps muscles to generate a peak force of 0.82 of MIF, an increase of $23 \%$ in the tibiofemoral contact force and a decrease in the 
patellofemoral contact force with respect to the healthy knee. These results show that $50 \%$ compensation is less demanding and also less damaging strategy for the knee than the total compensation.

\section{CONCLUSION}

The results of this study support results from previous studies $[10,11]$, indicating that an increase in the activation of hamstring muscles may compensate anterior tibial translation (ATT) in the ACLd knee. However, the present results add information indicating that, from comparing a $100 \%$ to a $50 \%$ compensation, a complete restoration of ATT to normal values during the whole gait cycle would require muscle training to achieve the forces required for knee stability and would cause an increase in the joint contact forces. A compensation of $50 \%$ of abnormal ATT, on the other hand, requires less force generated by the muscles and generates smaller joint contact forces, making it a less demanding and less deleterious strategy.

\section{REFERENCES}

[1] D. E. Hurwitz, T. P. Andriacchi, C. A. Bush-Joseph, and C. R. Bach, "Functional Adaptations in Patients with ACL-Deficient Knees," Exerc. Sport Sci. Rev., vol. 25, pp. 1-20, 1997.

[2] K. S. Rudolph, M. E. Eastlack, M. J. Axe, and L. Zinder-Mackler, "Movement Patterns after Anterior Cruciate Ligament Injury: a Comparison of Patients who Compensate Well for the Injury and Those who Require Operative Stabilization," J. Electromyogr. Kinesiol., vol. 8, pp. 349-362, 1998

[3] J. R. Steele, G. J. Roger, and P. D. Milburn, "Tibial Translation and Hamstring Activity during Active and Passive Arthrometric Assessment of Knee Laxity," Knee, vol. 1, no. 4, pp. 217, 1994.

[4] W. J. Hurd, and L. Snyder-Mackler, "Knee Instability after Acute ACL Rupture Affects Movement Patterns during the Mid-Stance Phase of Gait," J. Orthop. Res., vol. 25, pp. 1369-1377, 2007

[5] L. R. Osternig, B. L. Caster, and C. R. James, "Contralateral Hamstring (Biceps Femoris) Coactivation Patterns and Anterior Cruciate Ligament Dysfunction.," Med. Sci. Sports Excer., vol. 27, no. 6, pp. 805-808, 1995.

[6] G. Li, T. W. Rudy, M. Sakane, A. Kanamori, C. B. Ma, and S. L.Y. Woo, "The Importance of Quadriceps and Hamstring Muscle Loading on Knee Kinematics and In-Situ Forces in the ACL," $J$. Biomech., vol. 32, pp. 395-400, 1999.

[7] S. Hirokawa, M. Solomonow, Z. Luo, Y. Lu, and R. D’Ambrosia, "Muscular Co-Contraction and Control of Knee Stability," J. Electromyogr. Kinesiol., vol. 1, no. 3, pp. 199-208, 1991.

[8] A. Imran, and J. J. O'Connor, "Control of Knee Stability after ACL Injury or Repair: Interaction between Hamstrings Contraction and Tibial Translation," Clin. Biomech., vol. 13, no. 3, pp. 153, 1998.

[9] T. Yanagawa, K. B. Shelburne, F. Serpas, and M. G. Pandy, "Effect of Hamstrings Muscle Action on Stability of the ACL-Deficient Knee in Isokinetic Extension Exercise," Clin. Biomech., vol. 17, pp. 705-712, 2002.

[10] W. Liu, and M. E. Maitland, "The Effect of Hamstrings Muscle Compensation for Anterior Laxity in the ACL-Deficient Knee during Gait," J. Biomech., vol. 33, pp. 871-879, 2000.

[11] K. B. Shelburne, M. R. Torry, and M. G. Pandy, "Effect of Muscle Compensation on Knee Instability during ACL-Deficient Gait," Med. Sci. Sports Excer., vol. 37, no. 4, pp. 642-648, 2005.

[12] P. Klein, H. Schell, F. Streitparth, M. O. Heller, J.-P. Kassi, F. Kandziora, H. Bragulla, N. P. Haas, and G. N. Duda, "The Initial Phase of Fracture Healing is Specifically Sensitive to Mechanical Conditions," J. Orthop. Res., vol. 21, no. 4, pp. 662-669, 2003.

[13] A. Goodship, J. Cunningham, and J. Kenwright, "Strain Rate and Timing of Stimulation in Mechanical Modulation of Fracture Healing," Clin. Orthop., vol. 355, pp. 105 -115, 1992.
[14] P. P. Provenzano, D. A. Martinez, R. E. Grindeland, K. W. Dwyer, J. Turner, A. C. Vailas, and R. Vanderby, "Hindlimb Unloading Alters Ligament Healing.," J. Appl. Physiol., vol. 94, no. 1, pp. 314-324, 2003.

[15] D. Felson, "Weight and Osteoarthritis," J. Rheumatol., vol. 43, pp. 7-9, 1995.

[16] E. Abdel -Rahman, and M. S. Hefzy, "A Two-Dimensional Dynamic Anatomical Model of the Human Knee Joint," $J$. Biomech. Eng., vol. 115, pp. 357-365, 1993.

[17] D. A. Winter, Biomechanics and Motor Control of Human Movement, $3^{\text {rd }}$ ed. New Jersey: John Wiley and Sons, 2004.

[18] R. Nisell, G. Nemeth, and H. Ohlsen, "Joint Forces in Extension of the Knee," Acta Orthop. Scandinavica, vol. 57, pp. 41-46, 1986.

[19] K. B. Shelburne, and M. G. Pandy, "A Musculoskeletal Model of the Knee for Evaluating Ligament Forces during Isometric Contractions," J. Biomech., vol. 30, no. 2, pp. 163-17, 1997.

[20] G. T. Yamaguchi, and F. E. Zajac, "A Planar Model of the Knee Joint to Characterize the Knee Extensor Mechanism," J. Biomech. vol. 22, pp. 1-10, 1989.

[21] A. Garg, and P. S. Walker, "Prediction of Total Knee Motion Using a Three Dimensional Computer Graphics Model.," J. Biomech., vol. 23, no. 1, pp. 45-58, 1990.

[22] A. M. Ahmed, D. L. Burke, and A. Yu, "In Vitro Measurement of Static Pressure Distribution in Synovial Joints. Part II. Retropatellar Surface," J. Biomech. Eng., vol. 105, pp. 226-236, 1983.

[23] L. Blankevoort, J. H. Kuiper, R. Huiskes, and H. J. Grootenboer, "Articular Contact in Three-Dimensional Model of the Knee," $J$. Biomech., vol. 24, pp. 1019-1031, 1991.

[24] J. Wismans, F. Veldpaus, and J. Janssen, "A Three-Dimensional Mathematical Model of the Knee-Joint," J. Biomech., vol. 13, pp. 677-685, 1980 .

[25] N. D. Reeves, C. N. Maganaris, N. Maffulli, and J. Rittweger, "Human patellar Tendon Stiffness is Restored Following Graft Harvest for Anterior Cruciate Ligament Surgery," J. Biomech., vol. 42, pp. 797-803, 2009.

[26] S. L. Delp, "A Computer-Graphics System to Analyse and Design Musculoeskeletal Reconstruction of the Lower Limb", PhD Thesis, Standford University, Stanford, CA, USA, 1990.

[27] M. G. Pandy, and K. B. Shelburne, "Dependence of CruciateLigament Loading on Muscle Forces and External Load," $J$. Biomech., vol. 30, pp. 1015-1024, 1997

[28] S. T. Tumer, and A. E. Engin, "Three-Body Segment Dynamic Model of the Human Knee," J. Biomech. Eng., vol. 115, pp. 350356,1993

[29] C. L. Vaughan, B. L. Davis, and J. C. O'Connor, Dynamics of Human Gait. Cape Town, South Africa: Kiboho Publishers, 1999.

[30] D. A. Winter, Biomechanics and Motor Control of Human Movement, $2^{\text {nd }}$ ed. New York: John Wiley, 1990.

[31] M. P. Kadaba, H. K. Ramakrishnan, and M. E. Wootten, "Measurement of Lower Extremity Kinematics During Level Walking," J. Orthop. Res., vol. 8, pp. 383-392, 1990.

[32] J. Perry, Gait Analysis. Normal and Pathological Function. Thorofare, NJ: Slack Inc., 1992.

[33] M. W. Whittle, Gait Analysis. An Introduction. Oxford: Butterword-Heinemann, 2003.

[34] K. B. Shelburne, M. G. Pandy, F. C. Anderson, and M. R. Torry, "Pattern of Anterior Cruciate Ligament Force in Normal Walking," J. Biomech., vol. 37, no. 6, pp. 797, 2004.

[35] K. B. Shelburne, M. G. Pandy, and M. R. Torry, "Comparison of Shear Forces and Ligament Loading in the Healthy and ACLDeficient Knee during Gait," J. Biomech., vol. 37, no. 3, pp. 313, 2004.

[36] J. Kvist, and J. Gillquist, "Anterior Positioning of Tibia during Motion after Anterior Cruciate Ligament Injury," Med. Sci. Sports Exerc., vol. 33, no. 7, pp. 1063-1072, 2001.

[37] W. R. Taylor, M. O. Heller, G. Bergmann, and G. N. Duda, "Tibiofemoral loading during human gait and stair climbing," J. Orthop. Res., vol. 22, no. 3, pp. 625, 2004. 
[38] N. Zheng, G. Fleisig, R. Escamilla, and S. Barrentine, "An Analytical Model of the Knee for Estimation of Internal Forces During Exercise." J. Biomech., vol. 31, pp. 963-967, 1998.

[39] V. Baltzopoulos, "Muscular and Tibiofemoral Joint Forces during Isokinetic Concentric Knee Extension," Clin. Biomech., vol. 10, pp. 208-214, 1995.
[40] W. Liu, and M. E. Maitland, "Influence of Anthropometric and Mechanical Variations on Functional Instability in the ACLDeficient Knee," Ann. Biomed. Eng., vol. 31, pp. 1153-1161, 2003.

Received: December 28, 2009

(C) Catalfamo et al.; Licensee Bentham Open.

This is an open access article licensed under the terms of the Creative Commons Attribution Non-Commercial License (http://creativecommons.org/licenses/by-nc/3.0/) which permits unrestricted, non-commercial use, distribution and reproduction in any medium, provided the work is properly cited. 